\title{
Improving CT Image Analysis of Augmented Bone with Raman Spectroscopy
}

\author{
J. Charwat-Pessler, ${ }^{1}$ M. Musso, ${ }^{2}$ K. Entacher, ${ }^{1}$ B. Plank, ${ }^{3}$ P. Schuller-Götzburg, \\ S. Tangl, ${ }^{5,6}$ and A. Petutschnigg ${ }^{1}$ \\ ${ }^{1}$ University of Applied Sciences Salzburg, Markt 136a, 5431 Kuchl, Austria \\ ${ }^{2}$ University of Salzburg, Department of Materials Research and Physics, Hellbrunnerstraße 34, 5020 Salzburg, Austria \\ ${ }^{3}$ University of Applied Sciences Upper Austria, Stelzhamerstraße 23, 4600 Wels, Austria \\ ${ }^{4}$ Paracelsus Medical University Salzburg, Strubergasse 21, 5020 Salzburg, Austria \\ ${ }^{5}$ Medical University of Vienna, Karl Donath Laboratory for Hard Tissue and Biomaterial Research, Department of Oral Surgery, \\ Sensengasse 2a, 1090 Wien, Austria \\ ${ }^{6}$ Austrian Cluster for Tissue Regeneration, Donaueschingenstraße 13, 1200 Vienna, Austria
}

Correspondence should be addressed to J. Charwat-Pessler; johann.charwat-pessler@fh-salzburg.ac.at

Received 29 March 2013; Accepted 10 May 2013

Academic Editor: Hang Joon Jo

Copyright (C) 2013 J. Charwat-Pessler et al. This is an open access article distributed under the Creative Commons Attribution License, which permits unrestricted use, distribution, and reproduction in any medium, provided the original work is properly cited.

In recent years, bone graft substitutes have been increasingly used in the medical field, for example, in order to promote new bone formation. Microcomputed tomography $(\mu-\mathrm{CT})$ is an image-guided technique used in medicine as well as in materials science, enabling the characterization of biomaterials with high spatial resolution. X-ray-based methods provide density information; however, the question how far conclusions on chemical structures can be inferred from any kind of CT information has not been intensively investigated yet. In the present study, a bone sample consisting of autogenous bone derived cells (ABCs) and bovine bone mineral (BBM) was investigated by $\mu$-CT and Raman spectroscopic imaging, that is, by two nondestructive imaging methods. Thereby, the image data were compared by means of regression analysis and digital image processing methods. It could be found that $51.8 \%$ of the variance of gray level intensities, as a result of $\mu$-CT, can be described by different Raman spectra of particular interest for bone composition studies by means of a multiple linear regression. With the better description of $\mu$-CT images by the linear model, a better distinction of different bone components is possible. Therefore, the method shown can be applied to improve CT-image-based bone modeling in the future.

\section{Introduction}

Bone graft substitutes are widely used in the medical field and their usage covers a broad range of applications as for instance in dentistry or orthopedics. Bone grafting can be required in order to repair skeletal defects either to replace missing bone, filling voids, or to promote new bone formation so as to be able to place for instance an implant. In recent years an increase in demand for such bone graft substitutes could be observed [1-5] and due to the demographic situation especially in the United States and Europe a reversal of this trend is not forecast today. These bone substitutes encompass a variety of tissues and materials sources which can mainly be distinguished by their source and properties, respectively. In terms of their composition, bone graft substitutes can broadly be divided in autograft, allograft, and xenograft materials. Autograft materials are designated to a graft made of the body's own bone which is usually taken from the hip or ribs, while allograft materials are made of human tissues. Xenograft materials are derived from animals as cows or pigs and are in fact established as well; however, autograft materials are still considered as the gold standard in medicine for reasons of compatibility. Biomaterials can also be differentiated due to their properties as osteogenesis, osteoinduction, and osteoconduction. Osteogenesis describes the formation 
of new bone within the graft, osteoinduction refers to a stimulation of cells to convert into cells that are able to form bone, and osteoconduction means that the graft material acts as a scaffold that is ideally resorbed later on $[6,7]$. Due to the fact that autogenous bone is not always available in the required quantity, different materials have to be considered. The question how materials with respect to allograft and xenograft materials, respectively, behave after bone grafting in terms of bone regeneration mechanisms (i.e., osteogenesis, osteoinduction, and osteoconduction) and involved risks for the patient, is subject of current research in medicine [8-10]. The mechanical behavior after transplantation is a different question which is of central interest in the medical and engineering field and consequently subject of intense research efforts. Bone tissue and the associated mechanism of bone modeling as well as bone remodeling are not completely understood yet due to the complex hierarchical structure of that material $[11,12]$. Numerous different imaging techniques are used in order to address this problem. One of the probably most frequently used technologies in terms of clinical as well as material science is computed tomography (CT) and $\mathrm{X}$-ray microcomputed tomography $(\mu$-CT) whereby $\mu$-CT was developed based on computed tomography (CT) [13]. The benefit of $\mu$-CT is the higher spatial resolution and is considered as a powerful tool in medicine and bone research, respectively, $[14,15]$. In matters of micromechanical investigations, X-ray based technologies form the basis for $3 \mathrm{D}$ models that are used later for calculations based on finite elements [16-18]. Admittedly the information content of $\mu$-CT is strictly speaking limited to information of Xray absorption which does not necessarily allow conclusions on tissues, especially if two different materials show either a similar or nearly the same absorption coefficient. These established methods (i.e., CT, $\mu$-CT) are still part of science, but different techniques as Raman spectroscopy make an important contribution to the investigation of such previously mentioned questions [19-21], specifically as soon as Raman spectroscopy is used as imaging tool which is referred to as Raman spectroscopic imaging. Raman spectroscopic imaging can provide valuable clues in terms of the chemical structure, the molecular vibrations, and thus the chemical composition of cells and tissues, clues which are missing when regarding any kind of X-ray based method. Because that Raman spectroscopic imaging is bound by natural limitations on surfaces the implementation of the chemical information in generated $3 \mathrm{D}$ models requires as a prior condition the knowledge of how the relationship of these two different image data could be described in a mathematical way. Therefore the present study elaborates the question how far Raman spectra enable a description of a $\mu$-CT image by means of a multiple linear regression.

\section{Materials and Methods}

In the present study a bone sample consisting of autogenous bone derived cells (ABC) and bovine bone mineral (BBM) was investigated with two different imaging techniques whereas $\mathrm{ABC}$ as well as Bio-Oss were compounds of the appropriate sample [22]. Bio-Oss, produced by Geistlich Pharma AG, is a medical device based on bovine bone that is commonly used in bone regeneration due to its osteoconductive properties with respect to dental implants and periodontal defects, respectively. The sample originated from minipigs (i.e., a crossbred of Minnesota pigs and Vietnamese potbellied pigs) which were previously sinus grafted [22]. It was investigated by Raman spectroscopic imaging on the one hand and $\mu$-CT on the other hand. Because the comparison of the information from the two imaging methods is of central interest, the bone sample in the present study was cut with a saw so as to have a valuable point of reference when matching the images.

The $\mu$-CT data were collected by using a GE phoenix |Xray nanotom $180 \mathrm{NF}$ with a minimal possible isotropic voxel size (spatial resolution) of $0.5 \mu \mathrm{m}$. For the investigated bone samples, a voxel size of $9.79 \mu \mathrm{m}$ was chosen. The tomograms were generated with $80 \mathrm{kV}$ voltage at the nano focus tube, the measurement current was $300 \mu \mathrm{A}$, and the integration time at the detector was $500 \mathrm{~ms}$. Altogether 1.500 projections were recorded, which led to a total measurement time of about 90 minutes. For image reconstruction a filtered back-projection algorithm was applied by using the Nanotom reconstruction software datos $\mid \mathrm{X}$. The bone sample dried up to a moderate rate during the recording and was immediately put in an alcoholic solution again afterwards. This fact may have led to a certain degree of imprecision.

The confocal Raman based investigations were carried out with a Thermo Scientific DXR Raman microscope and a laser excitation at $780 \mathrm{~nm}$. The spatial resolution comprised an area of approximately 3 by $4 \mathrm{~mm}$ with an increment of $50 \mu \mathrm{m}$. The scanned area comprised an area of approximately 3 by $4 \mathrm{~mm}$ with a step size of $50 \mu \mathrm{m}$. In each measurement point the Raman spectrum was collected by accumulating 50 times with a period of 0.5 seconds for each single accumulation. Some of the recorded Raman spectra had a bad signal to noise ratio (SNR) because of fluorescence from impurities on the surface but were not removed from the data set due to data acquisition program limitations. As the acquisition time by confocal Raman spectroscopy lasted almost 50 hours, the bone sample, which was preserved in an alcoholic solution before the measurement procedure, was completely dried up after the Raman measurement, causing shrinkage of the sample.

Raman spectroscopic imaging results in a chemical map comprising a multitude of spectra and thus showing the intensities of the appropriate selected Raman shift. Raman bands of particular interest for compositional studies regarding bone are listed in Table 1 [21]. Each peak can be considered as an indicator for either bone, collagen, or other tissues. The two $\mathrm{CH}$ related vibrational modes are present in collagen as well as noncollagenous organic moieties. Bone apatite, in which phosphate is substituted among other with carbonate ions (i.e., type B carbonate substitution), changes various physical properties of hydroxyl(l)apatite (OHAp) and is used in bone graft substitutes due to its chemical similarity to bone mineral [23]. Carbonated hydroxy(l)apatite promotes the osseointegration of hydroxyl(1)apatite [24], but there are important chemical and structural differences between the 
TABLE 1: Selected Raman bands of bone and the manually defined maximum intensities.

\begin{tabular}{lccc}
\hline Raman peak & Peak position $\left(\mathrm{cm}^{-1}\right)$ & Intensity $(\mathrm{cps})$ & Presence of \\
\hline$v_{1} \mathrm{PO}_{4}$ & 961 & 400 & Bone \\
$v_{2} \mathrm{PO}_{4}$ & 438 & 200 & Bone \\
$v_{4} \mathrm{PO}_{4}$ & 589 & 200 & Bone \\
Amide I & 1677 & 150 & Collagen \\
Amide III & 1256 & 150 & Collagen \\
C-H bending & 1458 & 150 & Organic moiety \\
C-H stretching & 2937 & 500 & Organic moiety \\
Type B carbonate substitution & 1075 & 200 & Bone mineral \\
\hline
\end{tabular}

mineral in bone and carbonated apatite [23]. This should be taken into account regarding the indicated presence of type $\mathrm{B}$ carbonate substitution in Table 1. It could be found that the intensities of B type carbonate substitution concur extremely well with the Bio-Oss correlation map. Considering the fact that Bio-Oss is reported to contain carbonate apatite [25] the vibrational mode at $1075 \mathrm{~cm}^{-1}$ might explain most notably the presence of Bio-Oss as well.

In addition to these characteristic vibrational modes, reference measurements of known compounds were done in order to be able to compare the spectra of the bone sample with the spectra from a known compound. Correlation maps can help to identify different compounds in the augmented bone sample more easily which is part of the sample.

These materials mentioned previously were Bio-Oss, BioGide (a naturally resorbable bilayer membrane consisting of porcine collagen and produced by Geistlich Pharma AG), tricalcium phosphate powder (Carl Roth GmbH \& Co. KG) with a purity grade of $99.9 \%$, and bone as well as fat of pork, both provided by the local butcher.

The Raman spectra obtained with laser excitation at $780 \mathrm{~nm}$ showed a fluorescent background, which influences the quality of the data evaluation. All the spectra had therefore to be corrected applying an automatic baseline correction using the software solution of Thermo Scientific Inc. (OMNIC 8) for Raman applications.

There are a variety of ways to achieve a baseline correction using OMNIC 8. The basic idea is to fit a function through selected background points and subtract the resulting background from the trace. This can be achieved either by a linear equation or by polynomials of 1st up to 6th order. In order to find the coefficients of the function a matrix inversion technique is used. In case of logarithmic or exponential functions the equation is linearized prior to this by a natural log transformation. The underlying algorithm for an automatic baseline correction is commonly employed in industry in terms of spectral processing and fits the baseline through an iterative process [26].

On the basis of the chemical information contained in the Raman bands 13 different images could be generated, each showing the intensities related to the appropriate Raman band and reference (i.e., known compounds) spectrum, respectively. The key task of the present study was to determine how the different Raman spectra can describe the $\mu$-CT image on the basis of a linear relationship; therefore a multiple linear regression according to (1) was chosen for modeling:

$$
\widehat{Y}=b_{0}+b_{1} X_{1}+b_{2} X_{2}+\cdots+b_{i-1} X_{j-1}+b_{i} X_{j}
$$

With such a model it is not only possible to describe pure materials but also material compositions by means of Raman intensity spectra (term on the right side). These chemical data are used to describe the $\mu$-CT information (term on the left side). The regression parameters $b_{j}$ of the linear estimation function (1) are assumed by minimizing the residuals following the least square method in which each variable $X_{j}$ represents a Raman spectrum. For this purpose the $\mu$ CT image was considered as the dependent variable and the Raman spectra consequently as the independent variables. The constant $b_{0}$ can be interpreted as a gray level value from where all alterations work. In order to avoid redundant information in the model, variables (Raman spectra) that could be described by other variables within the data set were removed as soon as the coefficient of correlation exceeded a value of \pm 0.9 . The coefficients of correlation were calculated following Pearson's ratio which is defined as the covariance of two variables divided by the product of their standard deviation (2):

$$
\varrho(X, Y)=\frac{\operatorname{COV}(X, Y)}{\sigma(X) \sigma(Y)} .
$$

After the data set was screened with the help of a correlation matrix all those spectra which enabled a description of the $\mu$ CT image were determined. The odd data set (i.e., the data set without those spectra according to Table 2) formed the basis for a multiple linear regression. Additionally it can generally be specified that an unreasonable or unnecessary increase of explaining variables possibly leads to an artificially raised coefficient of determination of the model and thereby distorts the result. This fact emphasizes the need for data sets to be screened before performing a multiple linear regression.

The goodness of fit of the model can be verified on the one hand by means of the coefficient of determination and on the other hand by means of an F-test. If there is a true causal relationship between the dependent variables $X_{j}$ and the independent variable $\hat{Y}$, respectively, the coefficients $b_{i}$ are not equal to null. The null hypothesis suggests (3) that there 
TABLE 2: Correlations matrix of the Raman bands and the reference spectra following the order: (1) Bio-Oss, (2) tricalcium phosphate, (3) Bio-Gide, (4) pork bone, (5) pork fat, (6) Amide I, (7) Amide III, (8) $\mathrm{CH}$ bending, (9) $\mathrm{CH}$ stretching, (10) phosphate bending $\left(\nu_{2} \mathrm{PO}_{4}\right)$, (11) phosphate bending $\left(v_{4} \mathrm{PO}_{4}\right),(12)$ phosphate stretching $\left(\nu_{1} \mathrm{PO}_{4}\right)$, and (13) type $\mathrm{B}$ carbonate substitution.

\begin{tabular}{lcccccccccccccc}
\hline$i$ & 1 & 2 & 3 & 4 & 5 & 6 & 7 & 8 & 9 & 10 & 11 & 12 \\
\hline$(1)$ & 1 & 0.059 & -0.507 & -0.488 & -0.486 & 0.152 & 0.166 & 0.339 & -0.404 & -0.054 & -0.087 & 0.283 & 0.149 \\
$(2)$ & 0.059 & 1 & 0.562 & 0.680 & 0.335 & 0.251 & 0.140 & 0.055 & 0.396 & 0.620 & 0.556 & 0.804 & 0.482 \\
$(3)$ & -0.507 & 0.562 & 1 & 0.972 & 0.828 & 0.211 & 0.089 & 0.046 & 0.689 & 0.315 & 0.280 & 0.341 & 0.142 \\
$(4)$ & -0.488 & 0.680 & 0.972 & 1 & 0.841 & 0.246 & 0.120 & 0.067 & 0.740 & 0.440 & 0.398 & 0.468 & 0.261 \\
$(5)$ & -0.486 & 0.335 & 0.828 & 0.841 & 1 & 0.196 & 0.091 & 0.249 & 0.783 & 0.202 & 0.172 & 0.207 & 0.105 \\
$(6)$ & 0.152 & 0.251 & 0.211 & 0.246 & 0.196 & 1 & 0.957 & 0.850 & 0.555 & 0.737 & 0.746 & 0.579 & 0.838 \\
$(7)$ & 0.166 & 0.140 & 0.089 & 0.120 & 0.091 & 0.957 & 1 & 0.827 & 0.441 & 0.695 & 0.717 & 0.480 & 0.834 \\
$(8)$ & 0.339 & 0.055 & 0.046 & 0.067 & 0.249 & 0.850 & 0.827 & 1 & 0.497 & 0.464 & 0.470 & 0.411 & 0.636 \\
$(9)$ & -0.404 & 0.396 & 0.689 & 0.740 & 0.783 & 0.555 & 0.441 & 0.497 & 1 & 0.588 & 0.566 & 0.495 & 0.507 \\
$(10)$ & -0.054 & 0.620 & 0.315 & 0.440 & 0.202 & 0.737 & 0.695 & 0.464 & 0.588 & 1 & 0.985 & 0.793 & 0.923 \\
$(11)$ & -0.087 & 0.556 & 0.280 & 0.398 & 0.172 & 0.746 & 0.717 & 0.470 & 0.566 & 0.985 & 1 & 0.729 & 0.918 \\
$(12)$ & 0.283 & 0.804 & 0.341 & 0.468 & 0.207 & 0.579 & 0.480 & 0.411 & 0.495 & 0.793 & 0.729 & 1 & 0.783 \\
$(13)$ & 0.149 & 0.482 & 0.142 & 0.261 & 0.105 & 0.838 & 0.834 & 0.636 & 0.507 & 0.923 & 0.918 & 0.783 & 1 \\
\hline
\end{tabular}

is no difference between the regression coefficients $b_{i}$ of the function and can thus be rejected on the basis of an $F$-test:

$$
H_{o}: b_{1}=b_{2}=\cdots=b_{i}=0 \text {. }
$$

In this study two multiple linear regressions are presented. The first one, which refers later on to model 1 , incorporates all spectra whereas in the second one, which refers to model 2, variables not significant according to the $F$-test were excluded using the so-called backwards method. This technique operates progressively as it starts with a linear model including at first all variables and excludes variables afterwards step by step. This is done by evaluating the highest empirical $F$-value and has the benefit of reducing the amount of variables once more.

The coefficients $b_{i}$ of the hyperplane $\widehat{Y}$ (1) are not directly comparable according to their influence on the estimated variables as these explanatory variables (i.e., the independent variables) can be scaled differently. In order to be able to compare the explanatory variables with each other Backhaus et al. [27] suggests to standardize the coefficients following (4) where the so-called Beta values $\beta_{i}$ are the standardized coefficients, $b_{i}$ the unstandardized coefficients, $s_{x_{i}}$ the standard deviation of the appropriate variable, and finally $s_{y}$, which is the standard deviation of the dependent variable $\widehat{Y}$. The higher the influence of a variable, the higher the absolute value of the standardized coefficient:

$$
\beta_{i}=b_{i} \frac{s_{x_{i}}}{s_{y}}
$$

Initially the linear equation was calculated factoring all the remaining Raman spectra regardless whether the coefficients were significant with reference to a $t$-test or not. In a second stage the regression parameters $b_{j}$ were tested by means of a two tailed $t$-test at which the null hypothesis (5) was rejected

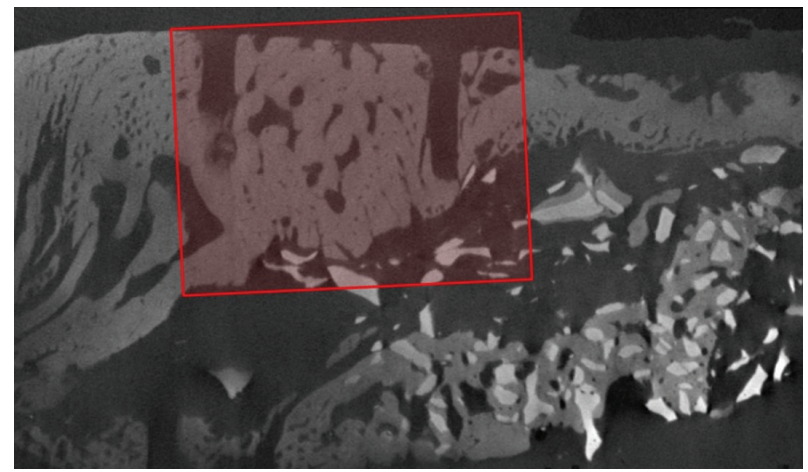

FIGURE 1: The appropriate region of interest related to the $\mu$-CT image.

in favor of the alternative hypothesis (6) at a confidence level of 0.95:

$$
\begin{aligned}
& H_{o}: b_{i}=0, \\
& H_{1}: b_{i} \neq 0 .
\end{aligned}
$$

It must be added that all calculations were carried out on the basis of gray level images although the Raman intensities in this paper are displayed in color. This does not have any impact on the results but solely on the perception of the information. Differences can be determined visually much more easily by the reader using color images instead of gray level images. All the charts and the associated statistical calculations were carried out in SPSS 19 whereas all the images were processed using MATLAB 2011a. In terms of a base line correction the spectra resulting from the Raman measurement were processed in OMNIC 8 as the algorithm for automatic base line correction was applied directly.

\section{Results}

The region of interest (ROI) is denoted by the red rectangle (Figure 1) in which the two kerfs serving as an orientation 
mark are located. Due to the fact that Raman spectroscopic imaging is limited to surfaces by natural conditions and $\mu$ CT provides images of the internal structure the very best $\mu$ CT image was selected manually being as close as possible to the surface and preserving an appropriate image quality at the same time.

The microscopic image in Figure 2(a) has been assembled from several individual images, which were stitched together to produce an overview image with high resolution. The squares are a result of this stitching process because the borders of the individual images remain visible. The accuracy of the microscopic evaluation is not affected by this phenomenon. In Figure 2 the microscopic image (Figure 2(a)) is compared with the corresponding Raman image (Figure 2(b)) showing the intensity distribution of the Amide III band, where red indicates a high and blue very low intensity.

The colors in between follow the order of the rainbow colors. In addition the Raman image was overlapped with the $\mu$-CT image (Figure 2(c)) in order to gain a first visual impression of the relationship between these two image data. In entirely practical terms it can be observed that the shrinkage as a consequence of dehydration of the bone sample due to the long term measurement is narrowed to a moderate rate. The generated Raman images (Figure 2(b)) turned out to vary in intensity to such an extent that some of the spectra were enhanced by reducing the maximum value of the range of intensity. This means that values which were at a low level before (f.i. green) are displayed subsequently at a higher level as orange or yellow for instance. Figure 3 shows in an exemplary manner the impact of such a processing. On the left side a chemical map focusing on a Raman peak at $1500 \mathrm{~cm}^{-1}$ is given. The intensity maximum of the range is indicated by OMNIC 8 with 420 . The maximum value was reduced in a second step to a value of 210. Lower intensities, which were poorly visible before (Figure $3(\mathrm{a})$ ) can now be determined in a better way (Figure 3(b)). At the same time the intensities at the green spot in the upper region are raised accordingly following the order of the rainbow colors. The maximum values, as they were set in the present study, are listed in Table 1.

In an exemplary manner the effect of the performed baseline correction is displayed in Figure 4 by means of the reference spectrum of pork bone. It can be seen how the background is obscured due to fluorescence and how the background is flattened by means of the baseline correction in virtue of the Thermo Scientific software solution for Raman applications.

In a further step all those spectra showing a coefficient of correlation greater than \pm 0.9 were removed from the data set and all further analyses were carried out with the reduced data set. In the event that highly correlated data within the data set could not be cleaned up the outcome will inevitably lead to a falsification of the explanatory linear model as explained in the previous chapter. In Table 2 the correlation matrix is given on which basis the data set was filtered.

Table 3 provides an overview of the spectra pairs exceeding the previously defined Pearson's correlation and those which were retained and removed respectively. It could be found that four data pairs fulfilled the determined criteria, accordingly four data set were removed and the statistical calculations were continued without Bio Gide, the Amide III band and without the two phosphate bending modes.

Table 4 sums up the results with model 1 referring to the forced multiple linear regression and model 2 referring to the regression eliminating all those coefficients having no significant contribution to the linear model according to the $t$-test. Furthermore the Raman peak at $438 \mathrm{~cm}^{-1}\left(v_{2} \mathrm{PO}_{4}\right)$ highly correlates with the Raman peak at $589 \mathrm{~cm}^{-1}\left(v_{4} \mathrm{PO}_{4}\right)$. Both were removed given that each of these Raman peaks highly correlates with the Raman peak at $1075 \mathrm{~cm}^{-1}$ and exceeded the previously determined criterion (i.e., \pm 0.9 ).

The global statistical evaluation shows by means of the coefficient of determination and the adjusted coefficient of determination, respectively, the goodness of fit of the total linear model. In conclusion at least $51.8 \%$ of the variation can be explained by the linear model. The goodness of fit remains unchanged after exclusion of the variable tricalcium phosphate and thus clearly indicates the very low explanatory amount of the same. All further observations are based on the multiple linear regression in which tricalcium phosphate was removed (model 2).

In Figure 5 the normal probability plot of the standardized residuals is given. In case of a perfect match all the data points would be on the linear equation. The residuals are arranged close to the straight line which emphasizes the normal distribution of the residuals. The assumption of normal distributed standardized residuals is relevant with reference to the coefficients (i.e., $b_{0}, b_{i}$ ) because these estimators are assumed to be normal distributed when choosing a multiple linear model and performing an $F$-test and $t$-test, respectively. In case of not normal distributed residuals the test statistics used would not be valid.

Table 5 gives an overview of the unstandardized coefficients as well as of the standardized coefficients and the appropriate $t$-test results for each linear model. The confidence interval is stated for the unstandardized coefficients.

The compound eliminated due to the $t$-test was the correlation map of tricalcium phosphate showing a significance of 0.593 and thereby exceeding the defined limit of 0.05 . Some of the values are low to such an extent that the decimal place cannot be seen in Table 5, but that does not mean that the significance has the value zero. As mentioned previously, the coefficients can be compared to each other directly as soon as they are standardized. The underlying intention thereby is to get rid of the different measurement dimensions which allow a comparison and thus a reasonable interpretation of these values with respect to their importance. In other words, the higher the value, the greater the importance of a variable $X_{i}$. A coefficient with a negative algebraic sign means that the constant (i.e., average gray level value) is decreased by the amount indicated by the appropriate coefficient $b_{i}$ and $\beta_{i}$, respectively. A positive coefficient means vice versa that the constant is increased by the correspondent coefficient $b_{i}$ within that linear model. However, the coefficients can only be interpreted that way if the $95 \%$ confidence interval 
TABLE 3: Spectra showing a correlation greater than \pm 0.9 .

\begin{tabular}{lccc}
\hline & Retained spectra & \multicolumn{2}{c}{ Removed spectra } \\
Raman peak & Peak position $\left(\mathrm{cm}^{-1}\right)$ & Raman peak & Peak position $\left(\mathrm{cm}^{-1}\right)$ \\
\hline Pork bone & Correlation map & Bio-Gide & Correlation map \\
Amide I & 1677 & Amide III & 1256 \\
Carbonate subst. & 1075 & $v_{2} \mathrm{PO}_{4}$ & 438 \\
Carbonate subst. & 1075 & $v_{4} \mathrm{PO}_{4}$ & 589 \\
\hline
\end{tabular}

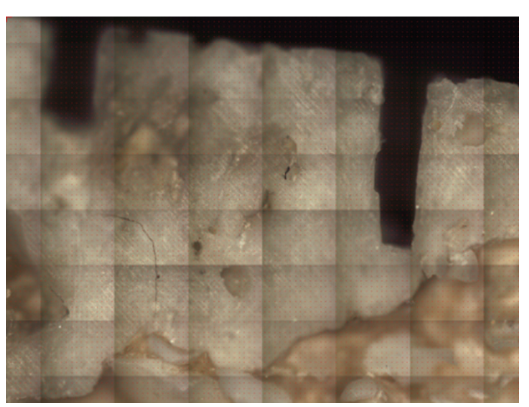

(a)

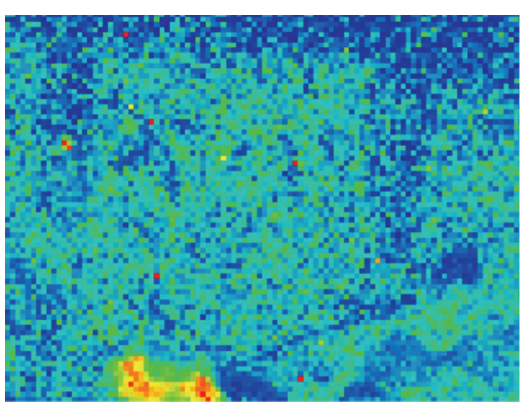

(b)

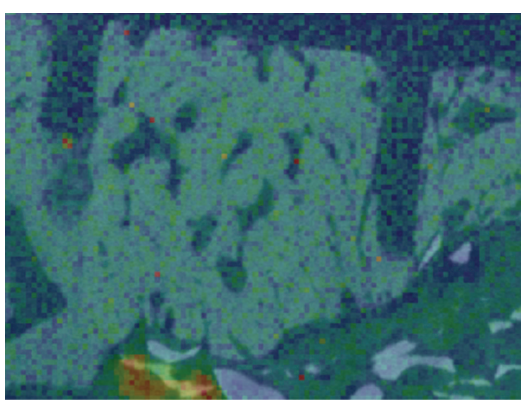

(c)

FIGURE 2: (a) Microscopic image, (b) Raman image showing the intensity distribution of the Amide III band, and (c) Raman image overlapped with the $\mu$-CT image.

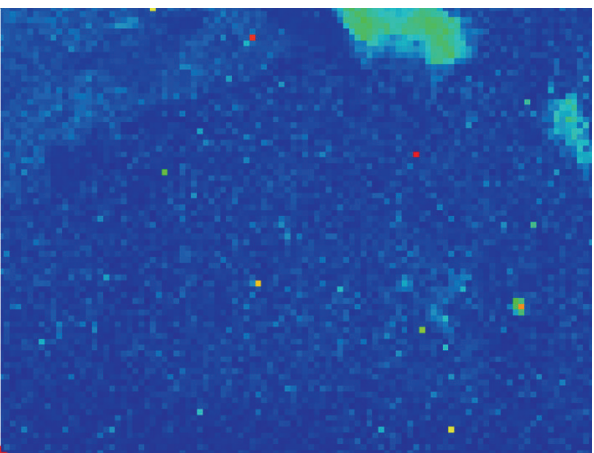

(a)

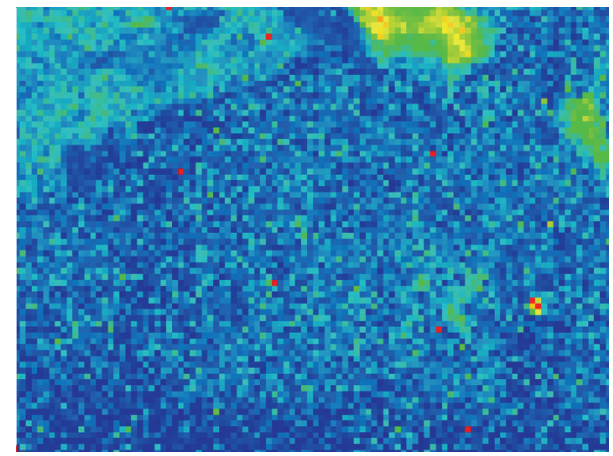

(b)

FIGURE 3: (a) Raman map with its original intensity range [0-420]. (b) Raman map with its reduced intensity range [0-210].

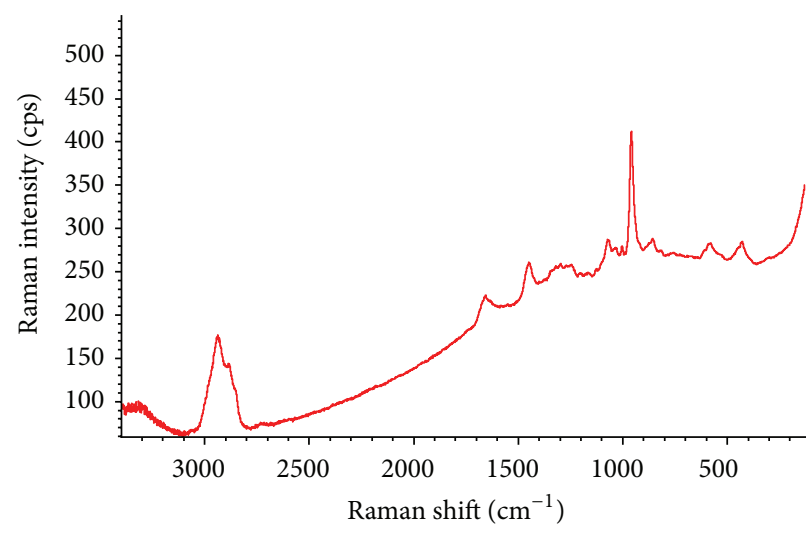

(a)

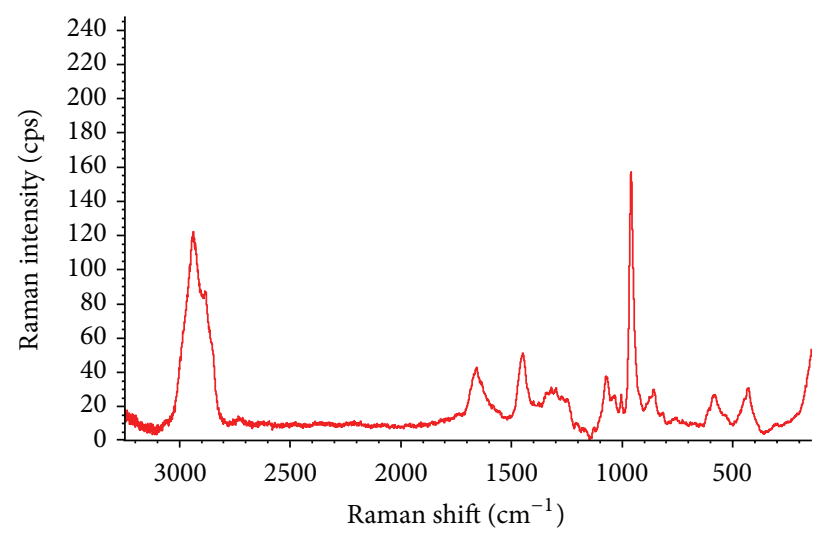

(b)

FIgURE 4: (a) Raman spectrum of pork bone showing fluorescence. (b) Corrected Baseline of the same spectrum. 


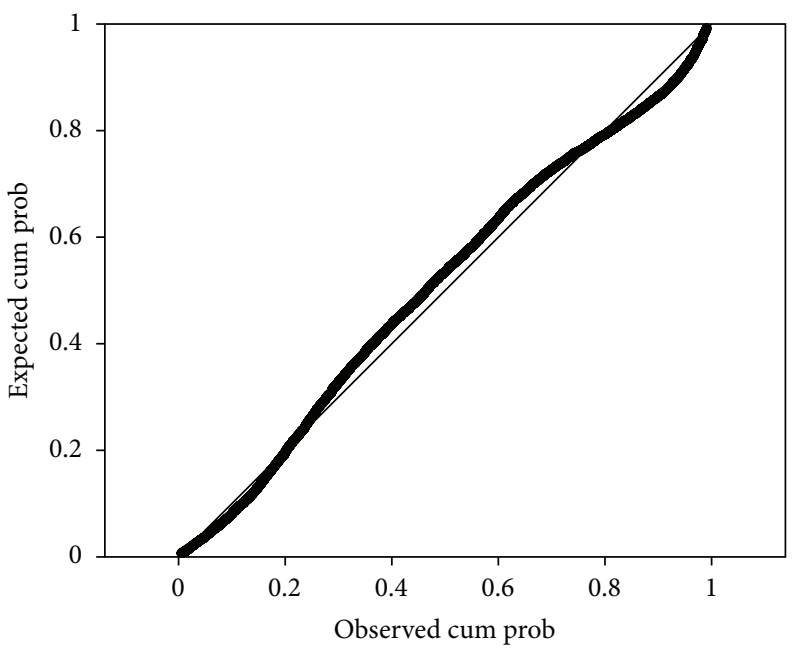

FIgURE 5: Normal probability plot.

TABle 4: Model summary.

\begin{tabular}{lccc}
\hline Model & $R$ & $R$ square & Adjusted $R$ square \\
\hline 1 & 0.720 & 0.519 & 0.518 \\
2 & 0.720 & 0.519 & 0.518 \\
\hline
\end{tabular}

does not include the value zero. As soon as zero is part of the interval this statement is not true. Furthermore the confidence intervals provide information with respect to the unstandardized coefficients.

Table 5 offers an overview of the standardized values of both linear models. It can be observed that the $\mathrm{CH}$ bending mode together with the pork fat correlation map is the strongest positive standardized estimators in model 1 and the only ones regarding model 2 . The confidence interval of these modes is positive as well and does not include the value zero for the unstandardized coefficients. This allows the conclusion that an increasing amount of $\mathrm{CH}$ bending mode and pork fat will lead to a higher $\mu$-CT value.

The most important negative estimators are given with the carbonate substitution mode and the correlation map of pork bone. The corresponding intervals are also negative and do not include the value zero. A higher amount of that mode and pork bone, respectively, will lead to a lower $\mu$-CT value. Such a breakdown (Table 5) shows which vibrational mode and component, respectively, influence the estimators (i.e., coefficient) the most.

In order to discuss the error of the presented linear regression model the residual plots are given in the form of scatterplots (Figure 6) in which the residuals of the regression model are plotted against each variable $X_{j}$. By means of these figures it can be determined whether the residuals are randomly scattered or not. If it happens that the residuals appear to be not randomly scattered, different additional parameters might be overlooked. Although tricalcium phosphate was excluded in model 2 the appropriate residual plot is nevertheless presented.
It can be observed that the scattering of the residuals appears differently in each case. Although the data seem to be well (i.e., randomly) spread around the red zero line, two main different data structures become visible and conspicuous, respectively, and are marked with two different colors (i.e., green and blue). In Figure 6(i) the intensity values for the type B carbonate substitution are displayed on the horizontal axis and the residuals are layed on the vertical axis. Those values which are framed with the green ellipse are distinctive and were accordingly assigned in the other figures (Figures 6(b), 6(c), 6(d), 6(f), and $6(\mathrm{~g}))$. These data points show an unreasonable SNR which might be caused by impurities on the surface of the bone sample [28].

In Figure 6(h) the intensity values for phosphate (Raman peak at $961 \mathrm{~cm}^{-1}$ ) and the residuals are displayed in exactly the same manner regarding the axes as previously described. The values which are marked with the blue ellipse in Figure 6(h) show an unreasonable SNR too. In large parts the values marked with the blue ellipse in Figures 6(e) and 6(f) are also affected by a bad SNR. A reason for this might be impurities on the surface of the sample again causing a general degradation of the Raman signal. This hypotheses, however, was not verified for which reason these data could not be excluded. These impurities might have changed their chemical composition due to the energy input of the laser which leads to the conclusion that any testing of that hypotheses is hardly feasible. Therefore a clear statement regarding these data would be too complicated.

Thus the error of the regression model can be traced back to two parameters. Firstly impurities on the surface of the sample are able to cause measurement errors which cannot be explained by the model and thus effect a major scattering. Secondly the model is not up to the task of explaining the overall variance. Different investigation methods could possibly lead to a clearer explanation regarding the material composition. 
TABLE 5: Results of the multiple linear regression.

\begin{tabular}{|c|c|c|c|c|c|c|}
\hline Model & Raman spectra & $\begin{array}{l}\text { Unstand. } \\
\text { coefficient }\end{array}$ & $\begin{array}{c}\text { Stand. } \\
\text { coefficient }\end{array}$ & $\begin{array}{c}\text { Significance } \\
(t \text {-test })\end{array}$ & \multicolumn{2}{|c|}{ Confidence interval (at a level of 0.95) } \\
\hline \multirow{10}{*}{1} & Constant & 182.438 & - & - & 165.405 & 199.471 \\
\hline & Bio-Oss & -0.050 & -0.049 & 0.022 & -0.092 & -0.007 \\
\hline & Tricalcium phosphate & 0.009 & 0.013 & 0.593 & -0.023 & 0.041 \\
\hline & Pork bone & -0.109 & -0.391 & 0.000 & -0.132 & -0.085 \\
\hline & Pork fat & 0.153 & 0.172 & 0.000 & 0.095 & 0.210 \\
\hline & Amide I & -0.087 & -0.064 & 0.058 & -0.176 & 0.003 \\
\hline & $\mathrm{CH}$ bending & 0.547 & 0.550 & 0.000 & 0.483 & 0.612 \\
\hline & $\mathrm{CH}$ stretching & -0.384 & -0.179 & 0.000 & -0.482 & -0.287 \\
\hline & Phosphate stretching & -0.099 & -0.201 & 0.000 & -0.126 & -0.071 \\
\hline & Carbonate substitution & -0.709 & -0.356 & 0.000 & -0.809 & -0.609 \\
\hline \multirow{9}{*}{2} & Constant & 183.728 & - & - & 167.366 & 200.090 \\
\hline & Bio-Oss & -0.048 & -0.047 & 0.026 & -0.090 & -0.006 \\
\hline & Pork bone & -0.104 & -0.375 & 0.000 & -0.122 & -0.087 \\
\hline & Pork fat & 0.147 & 0.165 & 0.000 & 0.094 & 0.199 \\
\hline & Amide I & -0.097 & -0.072 & 0.018 & -0.178 & -0.016 \\
\hline & $\mathrm{CH}$ bending & 0.551 & 0.554 & 0.000 & 0.488 & 0.614 \\
\hline & $\mathrm{CH}$ stretching & -0.389 & -0.181 & 0.000 & -0.485 & -0.293 \\
\hline & Phosphate stretching & -0.096 & -0.196 & 0.000 & -0.122 & -0.070 \\
\hline & Carbonate substitution & -0.701 & -0.352 & 0.000 & -0.797 & -0.605 \\
\hline
\end{tabular}

For this study a coefficient of correlation of 0.72 is sufficient in order to evaluate the presented method for which reason further methods do not appear necessary.

\section{Discussion and Conclusion}

Due to the fact that the sample was drying (moving) over time, for the applied $\mu$-CT scans a compromise of contrast, image quality and measurement time had to be found. Results according to this approach show that hardly any conclusion on the chemical composition of the sample can be made by means of a $\mu$-CT image solely. Alternatively to $\mu$-CT it may be possible to use synchrotron radiation for CT. Indeed, synchrotron radiation offers significant advantages in terms of coherence, brilliance, and divergence of the beam [29] which results in fewer artifacts, improved contrast, and resolution, as well as much lower measurement times. These aspects will be taken into account in further investigations since synchrotron radiation together with Raman spectroscopy might possibly make another important contribution to the characterization of biomaterials.

Raman spectroscopic imaging is a tool that is up to the task of providing valuable additional information and has been successfully used in the present study in order to characterize biomaterials such as bone samples consisting of bone graft substitutes.

The development and generation of the present mathematical model, comprising correlation calculations, multiple regression analysis, and digital image processing methods, showed that $51.8 \%$ of the variance of the gray level pixels can be successfully explained that way. Considering the fact that interpretations on the composition of bone graft substitutes and bone, respectively, are still carried out to a great extent on the basis of CT information, this study emphasizes the lack of deeper knowledge and understanding regarding the characterization of such materials on the one hand and highlights once more the importance of implementation of further imaging techniques on the other hand. Raman spectroscopy and other spectroscopic based examination methods should be embedded in further research efforts intensively since CT data do not provide reliable information on the chemical structure of biomaterials.

In view of the present results it can be stated moreover that this model needs to be improved by further data acquisition. The composition of the material in this study is very complex; thus further research efforts will be carried out on the basis of materials showing a lower degree of complexity with respect to their composition. The development of mathematical descriptions can thus be improved more easily in future.

This method is appropriate in order to improve the accuracy of 3D models which are exclusively based on CT image data and is thereby able to increase the quality of predictions on, for instance, micromechanical properties of bone and bone graft substitutes, respectively. An improved course of action in this regard is probably also of benefit to medical routines since the question how well such substitute materials are able to merge with body's own bone is still of importance in the medical field in terms of evaluation and characterization of such materials. 


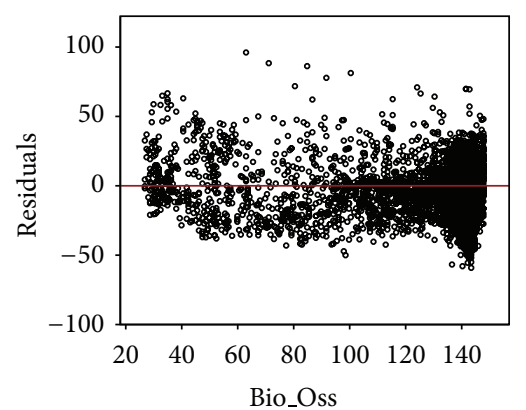

(a)

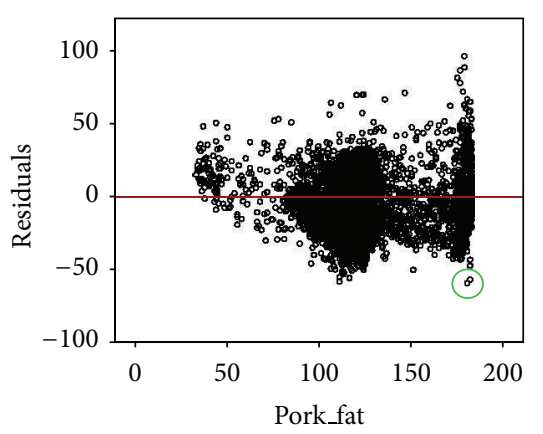

(d)

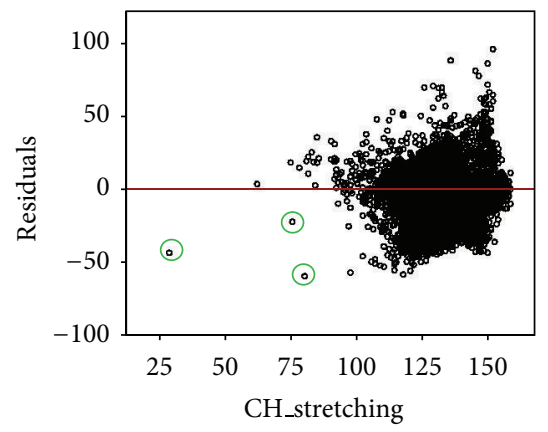

(g)

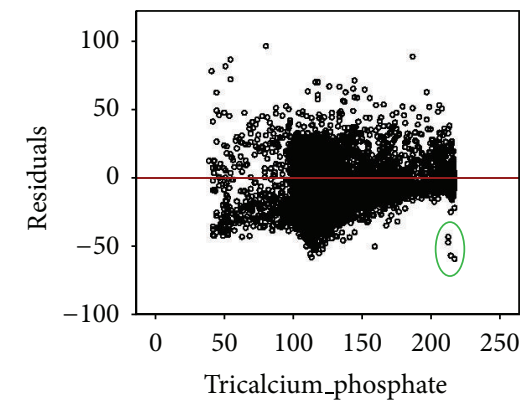

(b)

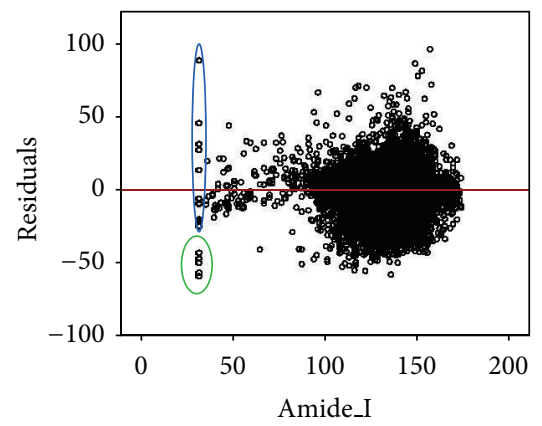

(e)

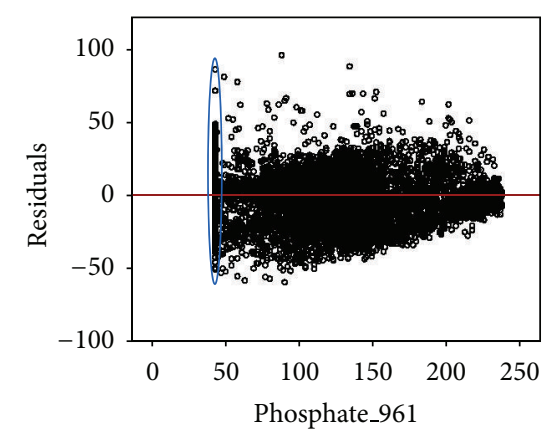

(h)

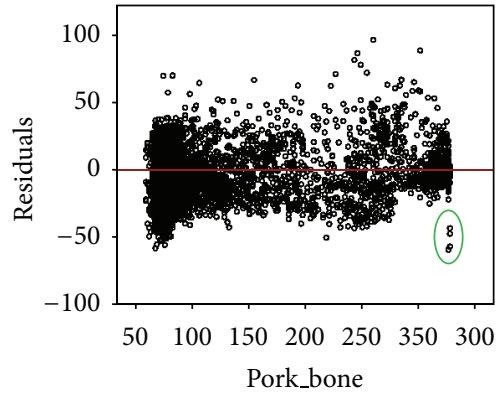

(c)

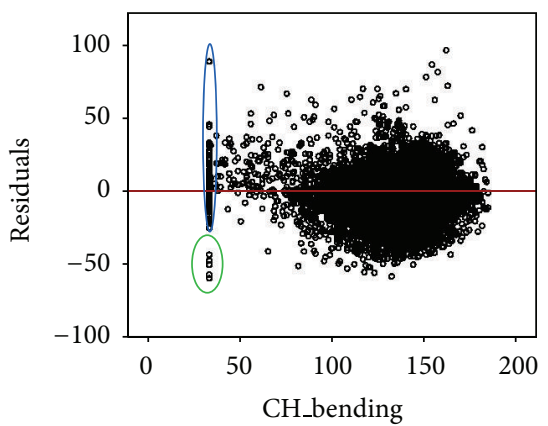

(f)

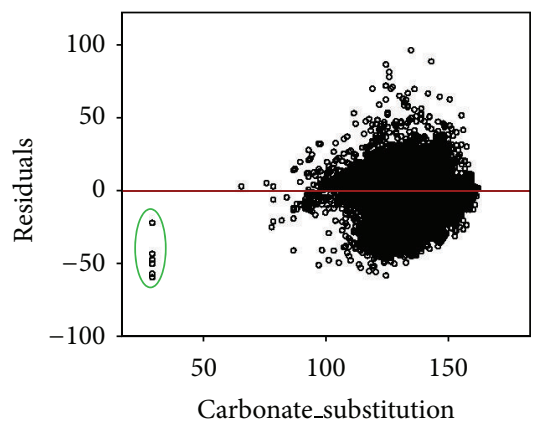

(i)

FIGURE 6: Residual plots and marked faulty measurements: (a) Bio Oss, (b) tricalcium phosphate, (c) pork bone, (d) pork fat, (e) Amide I, (f) $\mathrm{CH}$ bending, (g) CH stretching, (h) phosphate stretching at $961 \mathrm{~cm}^{-1}$, and (i) carbonate substitution.

The question as to how far chemical maps by means of Raman spectroscopic imaging are allowed or even should be processed in terms of digital image processing techniques remains open and should be generally discussed in a scientific context since every image enhancement possibly affects the result.

\section{Disclosure}

The authors have neither a direct nor an indirect financial relation with the commercial identity of the products mentioned in the present paper.

\section{Acknowledgments}

The authorship would like to thank the Austrian Science Fund FWF for financing the research project TRP 254N13. Furthermore the authorship would like to thank the
Medical University of Vienna, Department of Oral Surgery, for providing the bone sample.

\section{References}

[1] A. S. Greenwald, S. D. Boden, V. M. Goldberg, Y. Khan, C. T. Laurencin, and R. N. Rosier, "Bone-graft substitutes: facts, fictions, and applications," Journal of Bone and Joint Surgery A, vol. 83, supplement 2, pp. 98-103, 2001.

[2] C. Ogunsalu, "Bone substitutes and validation," in Implant Dentistry-The Most Promising Discipline of Dentistry, I. Turkyilmaz, Ed., InTech, New York, NY, USA, 2011.

[3] A. L. Dumitrescu, Chemicals in Surgical Periodontal Therapy, Springer, Heidelberg, Germany, 2011.

[4] A. S. Brydone, D. Meek, and S. Maclaine, "Bone grafting, orthopaedic biomaterials, and the clinical need for bone engineering," Proceedings of the Institution of Mechanical Engineers $H$, vol. 224, no. 12, pp. 1329-1343, 2010. 
[5] C. Laurencin, Y. Khan, and S. F. El-Amin, "Bone graft substitutes," Expert Review of Medical Devices, vol. 3, no. 1, pp. 49-57, 2006.

[6] L. S. Pryor, E. Gage, J. C. Langevin et al., "Review of bone substitutes," Cranial Maxillofac Trauma Reconstruction, vol. 2, no. 3, pp. 151-160, 2009.

[7] S. K. Nandi, S. Roy, P. Mukherjee, B. Kundu, D. K. De, and D. Basu, "Orthopaedic applications of bone graft \& graft substitutes: a review," Indian Journal of Medical Research, vol. 132, no. 7, pp. 15-30, 2010.

[8] W. R. Moore, S. E. Graves, and G. I. Bain, "Synthetic bone graft substitutes," ANZ Journal of Surgery, vol. 71, no. 6, pp. 354-361, 2001.

[9] X. Wang, Y. Zuo, D. Huang, X. Hou, and Y. Li, "Comparative study on inorganic composition and crystallographic properties of cortical and cancellous bone," Biomedical and Environmental Sciences, vol. 23, no. 6, pp. 473-480, 2010.

[10] F. Schwarz, K. Bieling, T. Latz, E. Nuesry, and J. Becker, "Healing of intrabony peri-implantitis defects following application of a nanocrystalline hydroxyapatite (Ostim) or a bovine-derived xenograft (Bio-Oss) in combination with a collagen membrane (Bio-Gide): a case series," Journal of Clinical Periodontology, vol. 33, no. 7, pp. 491-499, 2006.

[11] P. Fratzl, H. S. Gupta, E. P. Paschalis, and P. Roschger, "Structure and mechanical quality of the collagen-mineral nanocomposite in bone," Journal of Materials Chemistry, vol. 14, no. 14, pp. 2115-2123, 2004.

[12] P. Fratzl and R. Weinkamer, "Nature's hierarchical materials," Progress in Materials Science, vol. 52, no. 8, pp. 1263-1334, 2007.

[13] S. R. Stock, Microcomputed Tomography: Methodology and Applications, CRC Press, Boca Raton, Fla, USA, 2008.

[14] I. Kallai, O. Mizrahi, W. Tawackoli, Z. Gazit, G. Pelled, and D. Gazit, "Microcomputed tomographyg-based structural analysis of various bone tissue regeneration models," Nature Protocols, vol. 6, no. 1, pp. 105-110, 2011.

[15] H. D. Barth, E. A. Zimmermann, E. Schaible, S. Y. Tang, T. Alliston, and R. O. Ritchie, "Characterization of the effects of $\mathrm{x}$-ray irradiation on the hierarchical structure and mechanical properties of human cortical bone," Biomaterials, vol. 32, no. 34, pp. 8892-8904, 2011.

[16] A. Boccaccio, A. Ballini, C. Pappalettere, D. Tullo, S. Cantore, and A. Desiate, "Finite element method (FEM), mechanobiology and biomimetic scaffolds in bone tissue engineering," International Journal of Biological Sciences, vol. 7, no. 1, pp. 112132, 2011.

[17] G. Limbert, C. van Lierde, O. L. Muraru et al., "Trabecular bone strains around a dental implant and associated micromotionsA micro-CT-based three-dimensional finite element study," Journal of Biomechanics, vol. 43, no. 7, pp. 1251-1261, 2010.

[18] P. Schuller-Götzburg, K. Entacher, A. Petutschnigg, W. Pomwenger, and F. Watzinger, "Sinus lift with a cortical bone graft block: a patient-specific 3D finite element study," International Journal of Oral \& Maxillofacial Implants, vol. 27, no. 2, pp. 359-368, 2012.

[19] Y. Zhang, H. Hong, and W. Cai, "Imaging with Raman spectroscopy," Current Pharmaceutical Biotechnology, vol. 11, no. 6, pp. 654-661, 2010.

[20] M. D. Morris, M. V. Schulmerich, K. A. Dooley, and K. A. Esmonde-White, "Vibrational spectroscopic imaging of hard tissues," in Infrared and Raman Spectroscopic Imaging, R. Salzer and H. W. Siesler, Eds., pp. 149-171, Wiley-VCH, Weinheim, Germany, 2009.
[21] S. Gamsjäger, M. Kazanci, E. P. Paschalis, and P. Fratzl, "Raman application in bone imaging," in Raman Spectroscopy for Soft Matter Applications, M. S. Amer, Ed., pp. 227-267, John Wiley \& Sons, New Jersey, NJ, USA, 2009.

[22] G. Fuerst, S. Tangl, R. Gruber, A. Gahleitner, F. Sanroman, and G. Watzek, "Bone formation following sinus grafting with autogenous bone-derived cells and bovine bone mineral in minipigs: preliminary findings," Clinical Oral Implants Research, vol. 15, no. 6, pp. 733-740, 2004.

[23] B. Wopenka and J. D. Pasteris, "A mineralogical perspective on the apatite in bone," Materials Science and Engineering $C$, vol. 25, no. 2, pp. 131-143, 2005.

[24] A. Porter, N. Patel, R. Brooks, S. Best, N. Rushton, and W. Bonfield, "Effect of carbonate substitution on the ultrastructural characteristics of hydroxyapatite implants," Journal of Materials Science, vol. 16, no. 10, pp. 899-907, 2005.

[25] M. Figueiredo, J. Henriques, G. Martins, F. Guerra, F. Judas, and H. Figueiredo, "Physicochemical characterization of biomaterials commonly used in dentistry as bone substitutes-comparison with human bone," Journal of Biomedical Materials Research B, vol. 92, no. 2, pp. 409-419, 2010.

[26] "Thermo Scientific [homepage on the Internet]. Waltham, Massachusetts: Thermo Fisher Scientific Inc.(NYSE: TMO)," 2013, http://www.thermoscientific.de/ .

[27] K. Backhaus, B. Erichson, W. Plinke, and R. Weiber, Multivariate Analysemethoden: Eine Anwendungsorientierte Einführung, Springer, Berlin, Germany, 13th edition, 2011.

[28] R. L. McCreery, "Signal-to-noise in raman spectroscopy," in Raman Spectroscopy for Chemical Analysis, John Wiley \& Sons, Hoboken, NJ, USA, 2005.

[29] D. H. Bilderback, P. Elleaume, and E. Weckert, "Review of third and next generation synchrotron light sources," Journal of Physics B, vol. 38, no. 9, pp. S773-S797, 2005. 


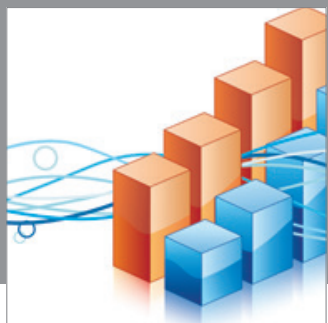

Advances in

Operations Research

mansans

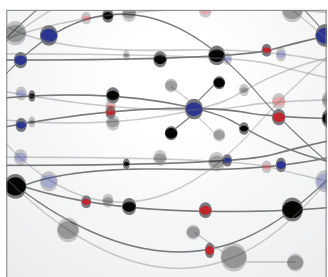

The Scientific World Journal
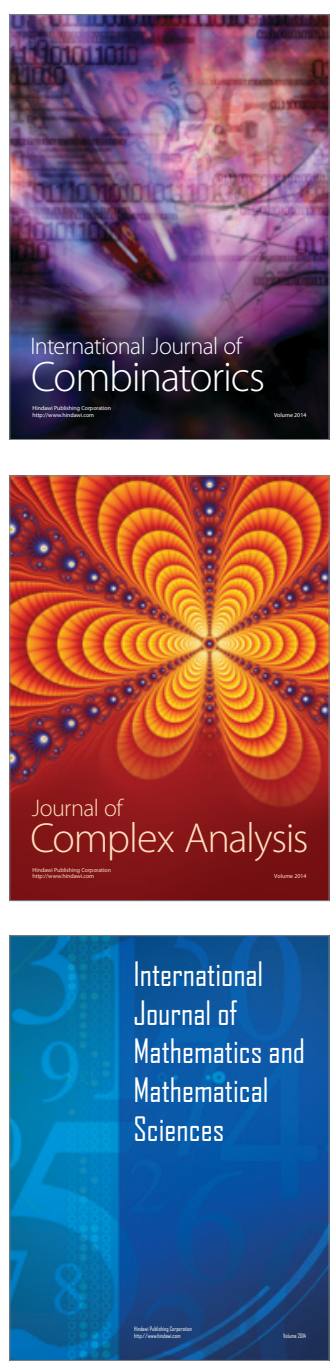
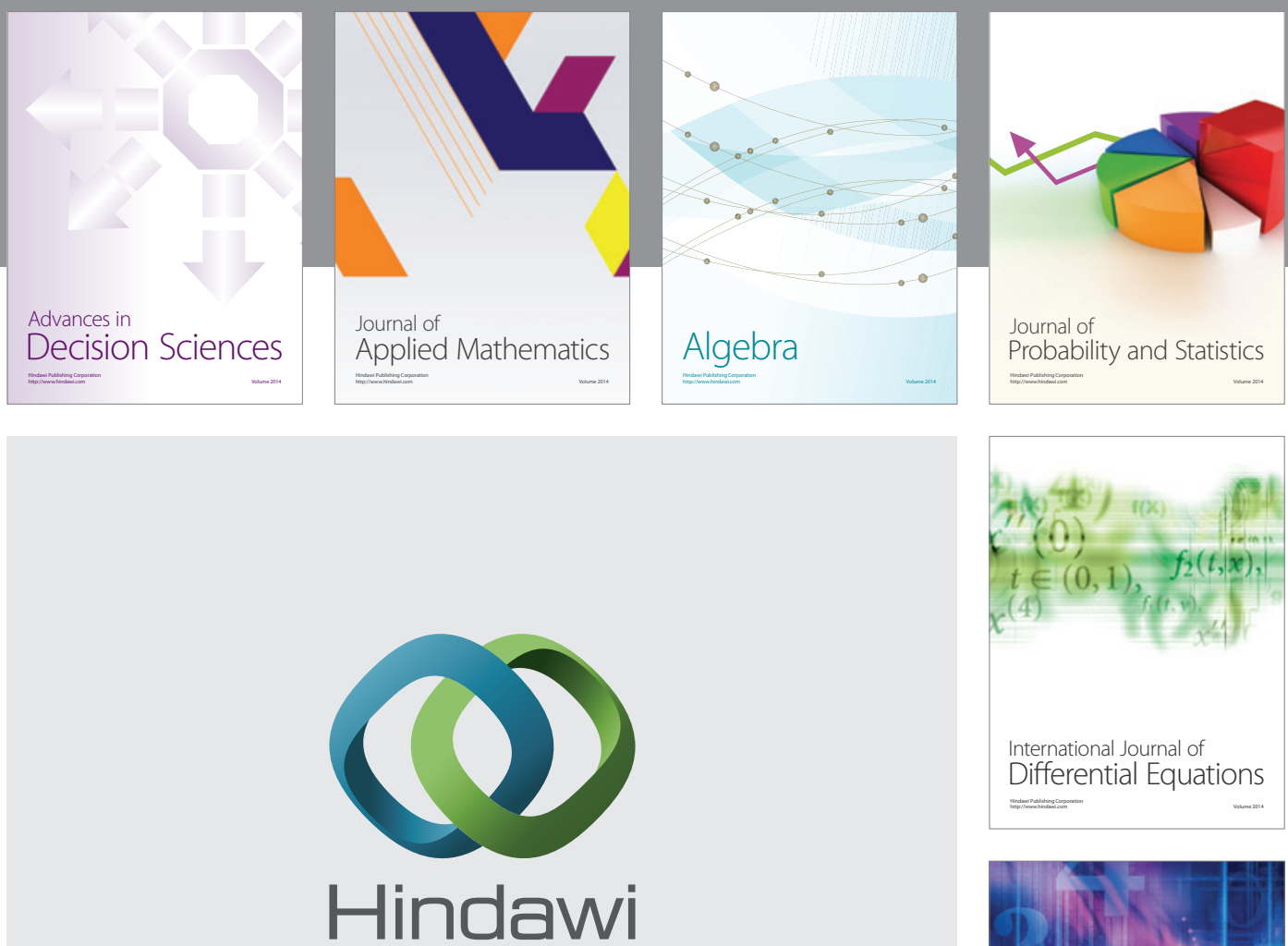

Submit your manuscripts at http://www.hindawi.com
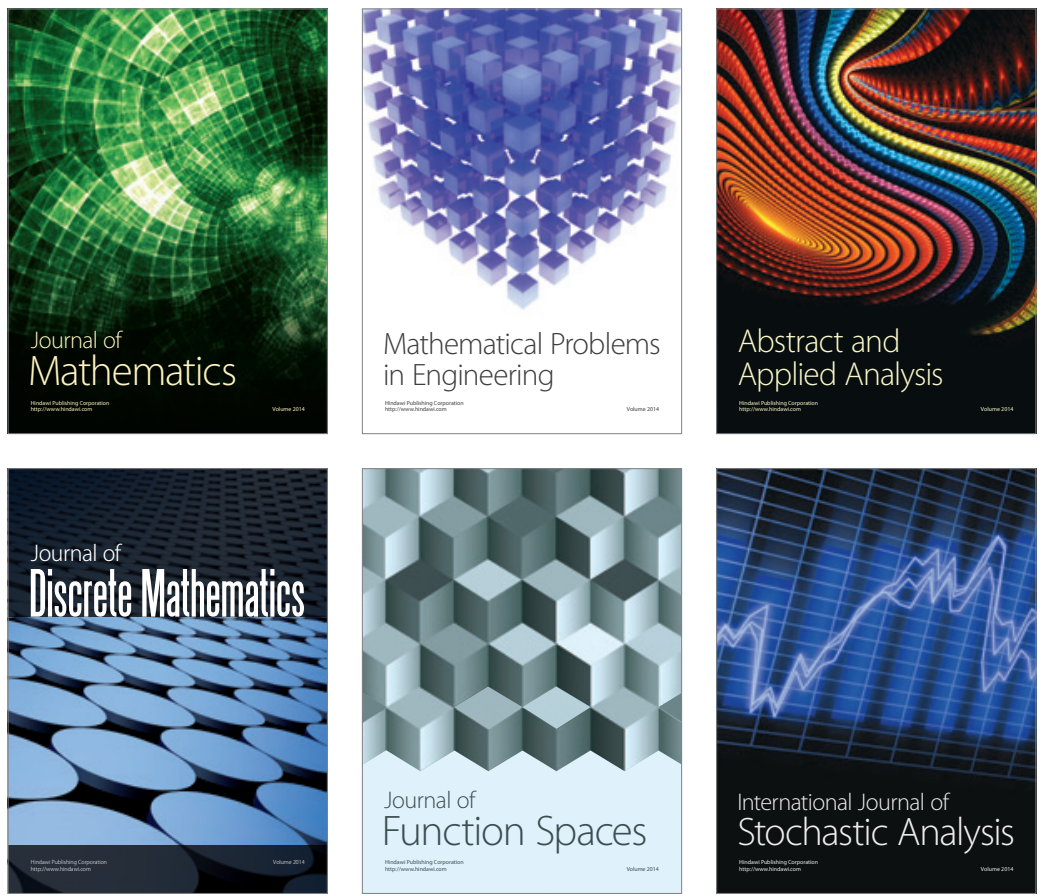

Journal of

Function Spaces

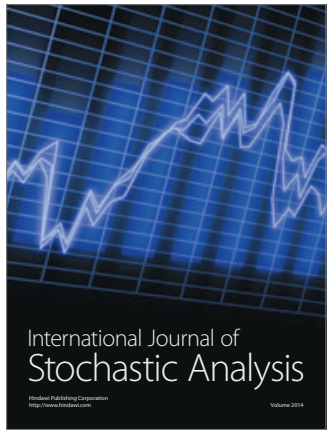

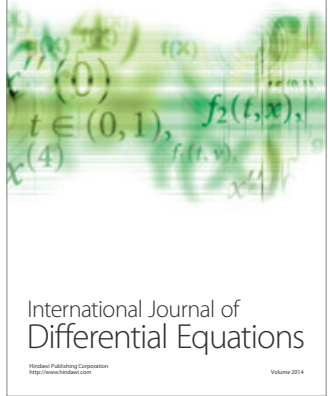
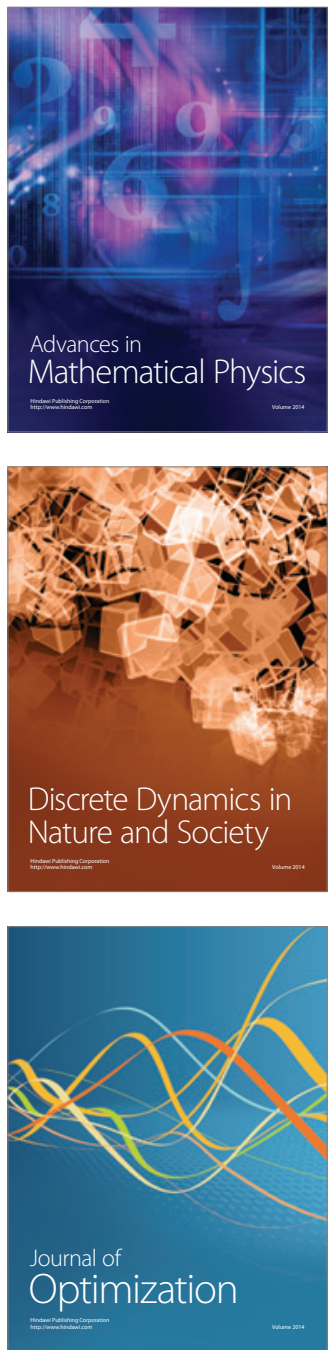